\title{
Pseudoaneurysma nach transradialer Punktion bei systemischer Lyse einer Basilaristhrombose
}

Michael-Ulrich Brehm, Kristina Sonnenschein, Jens Treptau, Thomas Aper, Johann Bauersachs, Muharrem Akin

Transradiale Punktionen werden immer häufiger durchgeführt. Nach dem Eingriff können Komplikationen wie das Pseudoaneurysma auftreten - so auch im Falle des hier vorgestellten Patienten. Obwohl eine Ultraschall-gesteuerte manuelle Kompression des Aneurysmas weniger Risiken birgt, entschieden sich die behandelnden Ärzte für eine chirurgische Resektion.

Anamnese I Ein 78-jähriger Patient $(167 \mathrm{~cm}$; $76 \mathrm{~kg})$ mit paroxysmalem Vorhofflimmern klagt über Gangunsicherheit, Anarthrie und Schwindel. In einem auswärtigen Krankenhaus wurde kürzlich eine erfolgreiche Kardioversion eines Vorhofflatterns durchgeführt. Das dort angefertigte Blutbild war unauffällig. Es gab keine Hinweise für eine Gerinnungsstörung. Als die neurologische Symptomatik begann, wurde der Patient rasch in unsere Klinik verlegt.

Thrombuslyse | Computertomografisch zeigt sich ein thrombotischer Verschluss der A. basilaris und der angrenzenden Aa. cerebri posteriores bds. Der Patient erhält eine Lysetherapie mit 86 mg Actilyse i. v. Im Anschluss steigen die Leukozyten vorübergehend auf max. 12400 / $\mu$ l an. Ein Infektfokus findet sich nicht.

Angiografie I Anschließend führen wir eine digitale Subtraktionsangiografie (DSA) der Kopf- und Halsgefäße mit Zugang über die A. femoralis communis links durch. Dabei findt sich eine offene

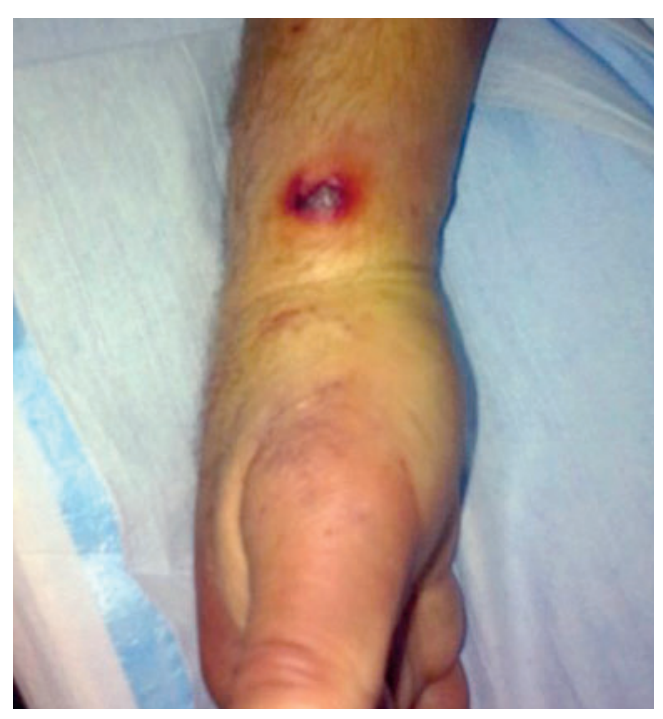

Abb. 1 Post-Lyse aufgetretenes Pseudoaneurysma der A. radialis rechts, 3 Tage nach Entfernung der Schleuse, die im Rahmen der invasiven Blutdruckmessung zur intensivmedizinischen Versorgung des Patienten angelegt wurde.
A. basilaris mit einem langstreckig umspültem Thrombus in der A. cerebri posterior P2 links. Der Patient hat einen Apoplex. Er wird intubiert auf der Intensivstation überwacht.

Transradiale Punktion | $2 \mathrm{~cm}$ oberhalb des Handgelenks punktieren wir die A. radialis rechts und legen einen 2,7 French großen arteriellen Zugang mit einer Länge von $8 \mathrm{~cm}$. Zur Steuerung der niedrigdosierten Katecholamintherapie erfolgt eine blutige Blutdruckmessung, die nach 5 Stunden beendet werden kann. Bald darauf wird der Patient komplikationslos extubiert. Der arterielle Zugang wird nach weiteren 16 Stunden entfernt, nachdem die hämodynamisch invasive Überwachung beendet ist. Aufgrund der systemischen Lysetherapie wird nach 10-minütiger manueller Kompression vorübergehend ein Kompressionsverband angelegt. Der Patient wird auf die Stroke Unit verlegt.

Körperliche Untersuchung I Zwei Tage später hat sich an der ehemaligen Punktionsstelle über der

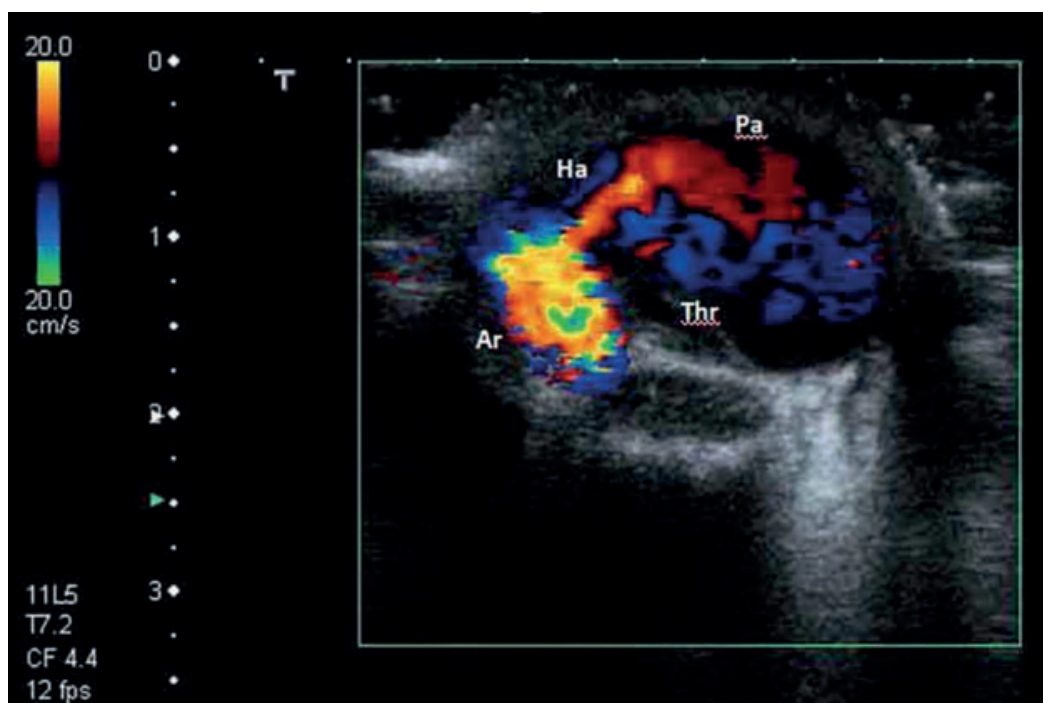

Abb. 2 Dopplersonografische Darstellung des Pseudoaneurysmas (Ps) der A. radialis (Ar) mit einer Ausdehnung von ca. $2 \mathrm{~cm}$, einer Thrombuslast (Thr) von ca. $20 \%$ und naher Lokalisation zum Aneurymsahals (Ha). 


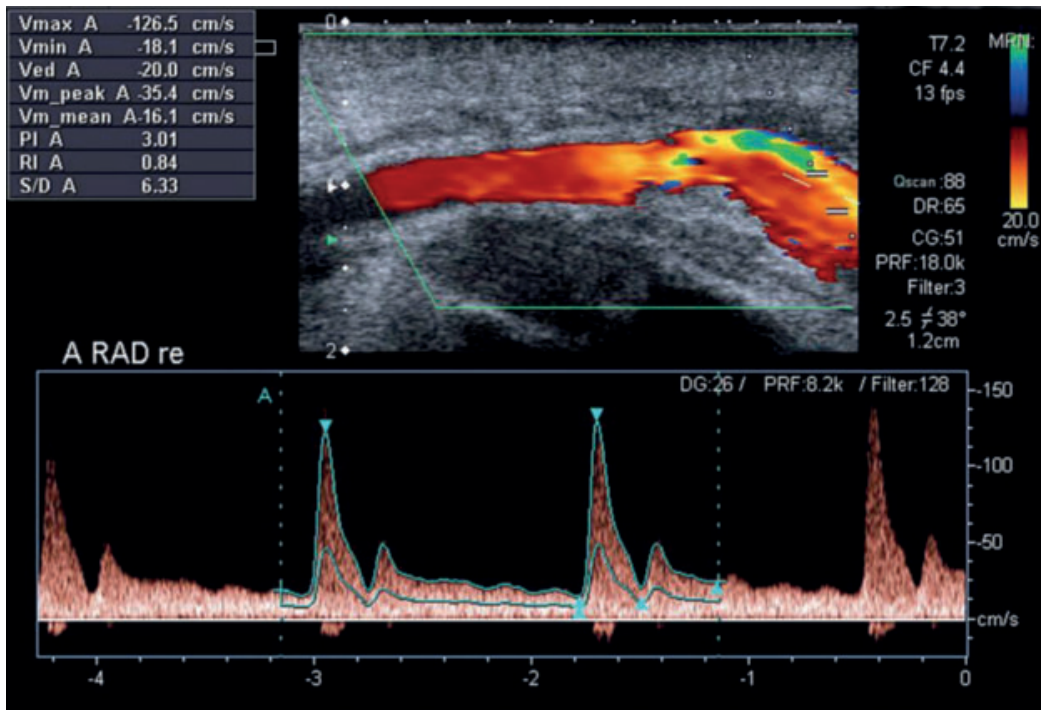

Abb. 3 Dopplersonografische Verlaufskontrolle der A. radialis 2 Tage nach operativer Resektion des Aneurysmasacks mit dicroter Pulswellenamplitude und ohne Hinweise auf ein Lokalrezidiv.

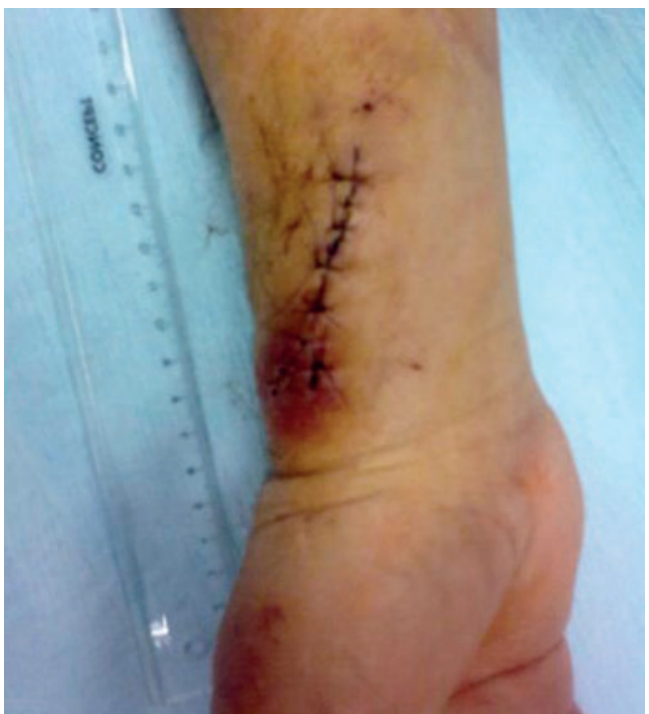

Abb. 4 Lokalbefund 2 Tage nach operativer Resektion des Aneurysmasacks.

sich ein biphasisches Signal ohne Alising und es liegt keine Flussbeschleunigung in der A. radialis und A. ulnaris vor. Insgesamt sind die Flussverhältnisse in der A. brachialis und A. subclavia normal. Der weitere klinische Verlauf ist unauffällig.

Laborbefunde I Prä-operativ und zum Zeitpunkt der Entlassung liegen die Leukozyten im Normbereich. Das CRP stieg zwischenzeitlich bis auf $31 \mathrm{mg} / \mathrm{l}$ an. Bei der Entlassung beträgt der CRPWert $9 \mathrm{mg} / \mathrm{l}$. Die Gerinnung ist bei einem Quick von $61 \%$ (INR 1,32) minimal. Zum Zeitpunkt der Operation lag der Wert bei 68\% (INR 1,23). Der Hb Wert war im Verlauf durchgehend im Normbereich.

Nachuntersuchung I Nach 3 Monaten bestätigt eine Kontrolluntersuchung eine regelrechte, reizlose und trockene Narbe über dem rechten Handgelenk ( Abb. 5). Motorik und Sensibilität der Hand und Finger sind weiterhin uneingeschränkt. Duplexsonografisch beträgt der Gefäßdiameter der A. radialis rechts $2,0 \times 2,8 \mathrm{~mm}$. Eine Striktur wird nicht nachgewiesen. Das biphasische Flusssignal liegt bei $58 \mathrm{~cm} / \mathrm{s}$.

\section{Diskussion}

Komplikationen beim Pseudoaneurysma I Ein Pseudoaneurysma wird auch als falsches Aneurysma oder Aneurysma spurium bezeichnet. Es entsteht durch eine Störung der Kontinuität der drei Wandschichten des arteriellen Gefäßes und beschreibt ein perivaskuläres, noch nicht vollständig thrombosiertes Hämatom mit anatomischer und hämodynamischer Verbindung zur Arterie. Dieses wird vom umliegenden Gewebe begrenzt, wobei eine Pseudowand aus Fibrin 


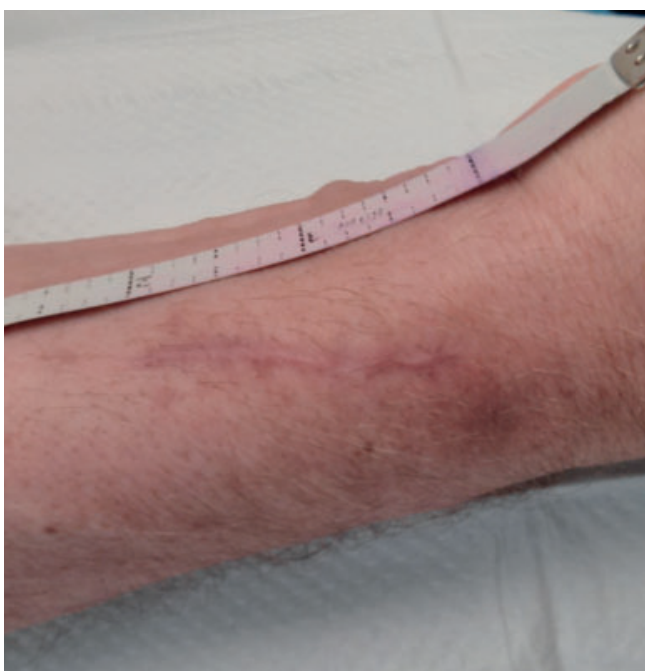

Abb. 5 Lokalbefund 3 Monate nach operativer Resektion des Aneurysmasacks und regelrechter Beweglichkeit, Motorik und Sensibilität der Hand (bis auf die Narbe).

entsteht. Pseudoaneurysmen der Unterarmarterien sind in der Regel gut zu beherrschen. Dennoch können folgende Komplikationen auftreten [2]:

- lokale Pulsationen

- Schmerzen der Hand

- thrombotische Verschlüsse der Handarterien

- motorische Schwäche oder neurologische Schädigungen der Hand

- Fingernekrosen mit Amputationsgefahr

Weiterhin besteht die Gefahr eines relevanten Blutverlustes bzw. der Ruptur des Pseudoaneurysmas.

Punktion der A. radialis | Die Anlage von Verweilkanülen erfolgt vor allem auf den internistischen und anästhesiologischen Intensivstationen bei intubierten und hämodynamisch instabilen Patienten mit Katecholaminbedarf - wie im beschriebenen Fall. Entsprechend den Empfehlungen der aktuellen Leitlinien der European Society of Cardiology (ESC) wird dieser Zugangsweg künftig auch vermehrt im Rahmen von Herzkatheteruntersuchungen gewählt werden [3]. Im Vergleich zu den relativ kleinen 3-French-Schleusen, die im Rahmen der Intensivüberwachung eingeführt werden, kommen hierbei zur Anwendung:

- 5-French-Schleusen für diagnostische Herzkatheteruntersuchungen

- 6-French-Schleusen für perkutan transluminale Koronarangioplastien (PTCA) in die A. radialis

Studienlage I In einer prospektiven Studie (The Leipzig prospective vascular ultrasound registry in radial artery catheterization) konnten bei transradial geführten Herzkatheteruntersuchungen mit überwiegend diagnostischer Koronarangiografie folgende Komplikationen beobachtet werden [4]:
- Verschluss der A. radialis

- Perforation

- Gefäßdissektion

- Aneurysma spuriae

Das Aneurysma spurium ist mit einer Rate von $0,2-0,4 \%$ eine seltene Komplikation [5, 6]. Nach klinischer Manifestation erfolgt durch die Duplexsonografie die Beurteilung der Lage, Größe und Ausdehnung von Hämatomen und dem Koagulationsstatus des Blutes in der Hämatomhöhle.

Therapie | Als therapeutische Maßnahmen stehen zur Verfügung:

- Ultraschall-gesteuerte manuelle Kompression des Aneurysmsas [7, 8]

- offen chirurgische Versorgung der Perforationsstelle mit Resektion der Aneurysmahöhle [9]

Die Thrombin-Injektion hingegen ist wegen des geringen Diameters des Pseudoaneurysmas der A. radialis und des kurzen Aneurysmahalses nicht zu empfehlen [10]. Welche Maßnahme ergriffen wird, hängt von mehreren Faktoren ab:

1. Risiko der Thrombusverschleppung in die Aa. metatarsale oder Aa. digiti

2. Thrombuslast im Aneurysmasack

3. Nähe der Thrombusmasse zum Aneurysmahals

4. Breite des Aneurysmahalses

5. Expertise vor Ort

Ultraschall-geführt oder operativ? | Bei der chirurgischen Versorgung können folgende Komplikationen auftreten:

- Narbenstrikturen

- Blutungen

- periphere arteriellen Embolien

- Gefäßrupturen

- Wundinfektionen

- Radikulopathien

- sekundäre Stenosierung der A. radialis

- relevante Sensibilitätsstörungen an der Nahtbzw. Narbenstelle

Daher bleibt diese Methode den klinisch instabilen Patienten und den großen Aneurysmata mit lokalen Komplikationen, wie einer ungünstigen Lokalisation der Thrombuslast im Aneurysma, vorbehalten. Das Ultraschall-gestützte Verfahren ist die effektivere und kostengünstigere Therapiemöglichkeit. Sie führt zu einer kürzeren Liegedauer der Patienten und zu weniger Langzeitkomplikationen. Bislang liegen für beide Methoden keine repräsentativen Daten zum Erfolg in großen Serien vor.

Entscheidung für die chirurgische Therapie | In unserem Fall haben wir wegen der ausgedehnten Thrombuslast und der Nähe des Thrombus zum Aneurysmahals die offene chirurgische Resektion der Aneurysmahöhle gewählt. Das Ziel war dabei, die Gefahr einer möglicherweise notwendigen zweiten Lysetherapie zu vermeiden. Denn aufgrund der bereits durchgeführten systemischen

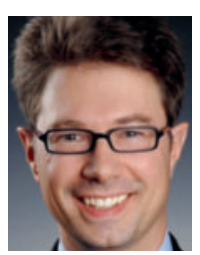

PD Dr.

Michael-Ulrich Brehm ist geschäftsführender Oberarzt an der Klinik für Kardiologie und Angiologie und Leiter der Angiologie, Medizinische Hochschule Hannover brehm.michael-ulrich@ mh-hannover.de

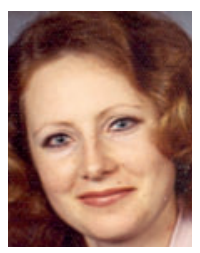

Dr. Kristina Sonnenschein ist Assistenzärztin an der Klinik für Kardiologie und Angiologie, Medizinische Hochschule Hannover sonnenschein.kristina@ mh-hannover.de

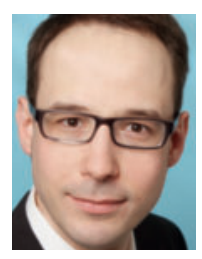

Dr. med. Jens Treptau ist Assistenzarzt an der Klinik für Kardiologie und Angiologie, Medizinische Hochschule Hannover treptau.jens@ mh-hannover.de 


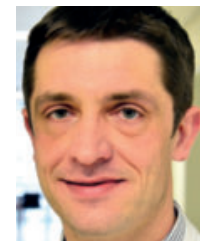

Dr. Thomas Aper ist Funktionsoberarzt für Gefäßchirurgie an der Klinik für Herz-, Thorax-, Transplantations- und Gefäßchirurgie, Medizinische Hochschule Hannover aper.thomas@ mh-hannover.de

Prof. Dr. Johann Bauersachs ist Direktor der Klinik für Kardiologie und Angiologie, Medizinische Hochschule Hannover bauersachs.johann@ mh-hannover.de

\section{Dr. Muharrem Akin} ist Assistenzarzt an der Klinik für Kardiologie und Angiologie, Medizinische Hochschule Hannover akin.muharrem@ mh-hannover.de
Lyse im Rahmen der Basilaristhrombose hätte es zur thrombembolischen Verschleppung und Verlegung der distal gelegenen Aa. metatarsale oder Aa. digiti kommen können.

Konsequenz für Klinik und Praxis

- Pseudoaneurysmen der A. radialis treten nach invasivem hämodynamischen Monitoring auf den Intensivstationen zunehmend häufiger auf. Ein Grund hierfür liegt in der Zunahme transradialer Punktionen im Rahmen diagnostischer Koronarangiografien sowie perkutanter Koronarangioplastien (PTCA) mit großlumigeren Schleusen als Zugangsweg.

- Als sichere Therapieoptionen stehen bislang die operative Resektion oder die Ultraschall-geführte manuelle Kompression zur Verfügung. Jedoch liegen derzeit keine größeren Vergleichstudien beider Methoden vor.

\section{Literatur}

1 Collins N, Wainstein R, Ward M et al. Pseudoaneurysm after transradial cardiac catheterization: case series and review of the literature. Catheter Cardiovasc Interv 2012; 80: 283-287

2 Rademakers LM, Laarman G]. Critical hand ischaemia after transradial cardiac catheterisation: an uncommon complication of a common procedure. Neth Heart J 2012; 20: 372-375
3 Hamon M, Pristipino C, DiMario C et al. Consensus document on the radial approach in percutaneous cardiovascular interventions: position paper by the European Association of Percutaneous Cardiovascular Interventions and Working Groups on Acute Cardiac Care and Thrombosis of the European Society of Cardiology. Eurolntervention 2013; 8: 1242-1251

4 Uhlemann M, Möbius-Winkler S, Mende M et al. The Leipzig Prospective Vascular Ultrasound Registry in Radial Artery Catheterization Impact of Sheath Size on Vascular Complications. J Am Coll Cardiol Intv 2012; 5: 36-43

5 Samaranayake CB, Timothy T], Stewart JT et al. Pseudoaneurysm of the radial artery following coronary angiography. Herz 2013; 8: 1-2

6 Bhat T, Bhat $\mathrm{H}$, Teli $\mathrm{S}$ et al. Pseudoaneurysm a rare complication of transradial cardiac catheterization: a case report. Vascular 2013; 21: 331-334

7 Nazer B, Boyle A. Treatment of recurrent radial artery pseudoaneurysms by prolonged mechanical compression. J Invasive Cardiol 2013; 25: 358-359

8 Liou M, Tung F, Kanei $Y$ et al. Treatment of radial artery pseudoaneurysm using a novel compression device. J Invasive Cardiol 2010; 22: 293-295

9 Igari K, Kudo T, Toyofuku T et al. Surgical Treatment of Aneurysms in the Upper Limbs. Ann Vasc Dis 2013; 6: 637-641

10 Kleczynski P, Rakowski T, Dziewierz A et al. Ultrasound-guided thrombin injection in the treatment of iatrogenic arterial pseudoaneurysms: Single-center experience. J Clin Ultrasound 2014; 42: $24-26$

\section{Interessenkonflikt}

Die Autoren geben an, dass kein Interessenkonflikt besteht.

DOI 10.1055/s-0041-107094

Dtsch Med Wochenschr 2016; 141: 211-214

(c) Georg Thieme Verlag KG . Stuttgart · New York .

ISSN 0012-0472 